\title{
Analysis of Key Generation Methods for Enable Secured Communication in the IoTs System
}

\author{
Nikhil Subhash Patankar', Dr. Parmalik Kumar², Dr. Rajneesh Karan ${ }^{3}$ \\ Ph.D. Scholar ${ }^{1}$, Professor ${ }^{2,3}$, Department of Computer Science \& Engineering ${ }^{1,2,3}$, Madhyanchal Professional \\ University ${ }^{1,2,3}$ \\ Bhopal, Madhya Pradesh, India
}

\begin{abstract}
The open stack communication protocol of the internet of things always in security threats. The vulnerability of protocol faces a problem of man in middle attacks and loss of integrity of communication. Cryptography plays a vital role in security authentication on IoT based enable communication system. IoT devices' limited resource constraints cannot offer any security mechanism on the physical level and network level. The key generation methods uplift the security provision in IoT enables communication model. The process of key generation applied three types of cryptography algorithm, symmetric, asymmetric and hybrid algorithm. The complexity of key generation methods needs more computational time and memory; this is the limitation of IoT devices. So various authors proposed a lightweight key generation algorithm for security and authentication. This paper analyzed the performance of key generation methods based on different cryptography approaches and measured their security strength. For the analysis process, use MATLAB software and sample text data file for the communication of two parties. Some ADHOC methods apply to measure protocol weakness during the communication network compromised with a man in the middle attack. The variable key size also reflects the security strength of IoTs enable devices.
\end{abstract}

Keywords: - Wireless Network, IoTs, Key Generation, Security, Authentication, Cryptography, Threats

\section{Introduction}

Scalability and integration of multiple devices with wireless sensor networks develop new communication horizons called the internet of things[1]. In the internet of things, many tiny devices are connected with the open stack communication protocol[2]. The applicability and diversity of IoTs enable communication in every filed of the real world such as medical instruments, smart home, smart transportation, smart agriculture and many more [3,
4]. The utility of communication devices needs a secured communication system, without threats and the possibility of data loss. The standard IoT devices are connected by wireless Wi-Fi, IEEE 802.15.4(ZigBee), Bluetooth, LoRa and Sigfox[5]. This communication medium is a week in concern of security, always come in the possibility of threats. The massive growth of IoT, there are many challenges and issue addressed with developing of IoT application. The connectivity range of IoT devices is extensive; every physical component heterogeneity connected with different standard protocol $[6,7,8]$. The mutual understating of the designing protocol is not common in every manufacture of IoT devices. The limited resource of IoT devices such as low energy, low bandwidth and low memory for the computational process's processing creates a vulnerability of security threats. Most IoT enables system integrates security mechanisms within wireless sensor networks to provide efficient security for their application. Generally, symmetric cryptography and hash function are applied. The asymmetric cryptography takes more memory for the computational key and session, avoid this for integration. The strength of security measurements if week due to the same code of authentication and identification $[9,10]$. The two major issues in concern of IoT enable communication is authentication and secure transmission of data. The authentication process verifies the identity of users [11, 12]. It prevents the network with a malicious user from accessing the network- the conventional security mechanism based on sharing the same key for communication and authentication. The same code's nature is fragile and easily tamper by third parties and loses integrity and confidently of communication and data, even though various security models of communication in IoT enabled device. The cryptography plays a leading role in the generation of key and distribution of the key[13]. The major factor of the cryptography algorithm is a complex formulation of the mathematical derivation. The applied derivation needs much more time and memory for the processing of the generation process. The major security attacks of IoT enable device related to the wireless sensor network [14, 
15]. The wireless sensor network compromised mainly wormhole attack, black hole attack, impression attack, noise attack and DDOS attacks. These types of attack theft the information during the communication period, but the user cannot fill the data leakage. Such types of attack deal with different security protocols and cryptography algorithms and reduce security threats. Some authors and research scholar applied the signal based vital generation process for communication- the signal based key generation methods such as DCT and DWT $[16,17]$. The generated required methods are very efficient and provide great concern for security but face a limited bandwidth of transmission. Despite many

\section{RELATED WORK}

The security is major issue in the aera of internet of things. For the management of security and key generation process applied various protocol and cryptography algorithm. Some authors describe the methods of key generation and authentication process. The contribution of work is described in the way of entitle here. Khalid, Umair Et al. [1] a decentralized affirmation and access control framework is discussed for lightweight IoT contraptions and is appropriate to a gigantic number of circumstances. The segment relies upon the development of the fog enrolling and the possibility of the public blockchain. The results procured from the tests display a dominating introduction of the discussed framework when appeared differently in relation to a top tier blockchain-based confirmation technique. Sobin, C. C. Et al. [2] authors have surveyed various architecture and protocols used in IoT systems and discussed suitable taxonomies for classifying them. authors have also discussed the technical challenges, such as security and privacy, interoperability, scalability, and energy efficiency. authors have provided an in-depth coverage of recent research works for every mentioned challenge. The objective of this survey is to help future researchers to identify IoT specific challenges and to adopt appropriate technology depending on the application requirements. Cao, Jin Et al. [3] a quantum resistance access authentication and data distribution scheme are discussed for large-scale NB-IoT devices. The scheme can simultaneously implement access authentication and data transmission of a group of NBIoT devices based on the lattice-based homomorphic encryption technology. Their scheme not only greatly reduce the network burden, but also achieve strong security including privacy protection and anti-quantum attacks. The performance analysis results show that their scheme has the ideal efficiency. Chen, Bin Et al. [4] creators focus in on arranging an amazing improvement protocol and algorithms for the secured communication in IoT enabled devices. Cryptography algorithms play an important role[18]. This paper focuses on the role of all types of cryptography algorithms in the process of analysis. The primary focus on public and private key generation process. The generation process of key measure the computational complexity and desirability of devices for the mode of communication. The rest of the paper organized as in section II. Describe the related work in the area of IoT. In section III. Describe the security threats and approach of cryptography, in section IV. Experimental analysis of key generation methods and finally conclude in section $\mathrm{V}$.

to get secret keys from SRAM-PUFs, which appreciate the uniqueness and assertion properties starting from the amassing assortments of SRAM memory cells. makers use a polar code improvement. Considering the way that SRAM memory can routinely be found in the present IoT contraptions, and since polar codes have been picked as goof amendment strategy in the 5G standard, this makes the analyzed plot a promising opportunity for decreasing the extra cost and ensuring about resource constrained IoT devices. Makers discussed a novel improvement technique to shed the effect of disturbance and tendency in SRAM-PUFs. makers will show that the secret spillage of the associate data about the secret key can be made insignificant as a result of polarization and authentic code improvement plan. Results show that the discussed plan gives a colossal improvement of the faithful quality and of the possible secret key rate, which is also evaluated by the theoretical assessment. Additionally, the discussed plan gives the probability to bargain multifaceted nature, secret and unfaltering quality with a comparative code improvement for different IoT applications. Deng, Lianbing Et al. [5] makers explore the characteristics of frameworks organization security and security issues, and analyze the system construction of Internet security and some key security developments, including key organization, check and access control, controlling security, security protection, interference acknowledgment and variation to non-basic disappointment and interference, etc. This paper presents the recent concerns of IoT in association security, and points out the need of interference disclosure. A couple of kinds of interference ID developments are analyzed, and its application on IoT designing is dismembered. makers take a gander at the utilization of different interference acknowledgment progressions, and make a chance of the accompanying time of investigation. Using data mining and AI methodologies to analyze network interference development has become a hot issue. A lone class feature 
or an acknowledgment model is very difficult to improve the disclosure speed of association interference area. The presentation of the inspected model is endorsed through the public data bases. Fan, Kai Et al. [6] authors discussed an efficient and privacy preserving outsourced multi-authority access control scheme, named PPOMACS. All attributes of users are transformed to be anonymous and authenticable to realize privacy preserving. And the verifiable outsourced decryption is introduced to reduce computation overheads on the end user side. Meanwhile, an efficient user revocation method is discussed. Security and performance analysis show that their scheme is secure and highly efficient. Hamza, Rafik Et al. [7] authors discussed a privacy preserving chaos-based encryption cryptosystem for patients' privacy protection. The discussed cryptosystem can protect patient's images from a compromised broker. In particular, authors discussed a fast probabilistic cryptosystem to secure medical keyframes that are extracted from wireless capsule endoscopy procedure using a prioritization method. The encrypted images produced by their cryptosystem exhibits randomness behavior, which guarantee computational efficiency as well as a highest level of security for the keyframes against various attacks.

Lee, Joohee Et al. [8] Authors examined the RLizard KEM, whose security relies upon the ring learning with mistakes and ring learning with adjusting issues. Since RLizard works on an exceptional sort of ring, it is more efficient as far as both the clock cycles needed for key age and the key size contrasted and the first Lizard conspire. To exhibit the prevalence of the talked about strategy over other notable KEMs, creators analyzed their exhibitions in the 32-digit ARM IoT climate. The presentation investigation indicated that the RLizard KEM requires the least clock cycles for key age, exemplification, and decapsulation when the boundaries are set to help a security level tantamount with that of AES-128. In synopsis, the RLizard KEM is relied upon to be utilized for secure correspondence and validation between IoT endpoint gadgets, whose computational force is for the most part restricted. Nausheen, Farha Et al. [9] the advantages of medical care IoT framework and the potential weaknesses that may result are introduced. Additionally, creators examined to create arrangements against these weaknesses by securing portable applications utilizing jumbling and return situated programming strategies. These procedures convert an application into a structure which makes hard for an enemy to decipher or adjust the code for ill-conceived reason. The portable applications use keys to control correspondence with the implantable clinical gadgets, which should be ensured as they are the basic part for making sure about interchanges. Thusly, creators likewise examined admittance control plans utilizing white box encryption to make the keys undiscoverable to programmers. Qu, Chao Et al. [10] to establish the relationship between IoT and $\mathrm{BC}$ for device credibility verification, authors discussed a framework with layers, intersect, and self-organization BCS. In this new framework, each BCS is organized by Blockchain technology. authors describe the credibility verification method and show how it provide the verification. The efficiency and security analysis are also given in this paper, including its response time, storage efficiency, and verification. The conducted experiments have been shown to demonstrate the validity of the discussed method in satisfying the credible requirement achieved by Blockchain technology and certain advantages in storage space and response time. Shah, Trusit Et al. [11] authors present a multi-key based mutual authentication mechanism. In their approach, the shared secret between the IoT server and the IoT device is called secure vault, which is a collection of equal sized keys. Initial contents of the secure vault are shared between the server and the IoT device and contents of the secure vault change after every successful communication session. authors have implemented this mechanism on an Arduino device to prove their algorithm is feasible on IoT devices with memory and computational power constraints. Song, Yujiao Et al. [12] authors design a new ABE scheme that protects users privacy during key issuing. In their new scheme, authors separate the functionality of attribute auditing and key generating to ensure that the KGC cannot know users' attributes and that the AAC cannot obtain the users secret key. This is ideal for many privacy-sensitive scenarios, such as industrial big data scenario. Soni, Ankit Et al. [13] creators talked about a remote mystery key age approach by pre-handling the RSSI of signals traded among Alice and Bob utilizing MWA. Their examined approach has critical improvement in execution, at lower SNR range. The size of the moving window is chosen based on progress in the standard deviation of the RSSI design. It is likewise proposed to quantize the pre-handled RSS tests through Lloyd'Max based quantizer to limit quantization mistake, which further improves bit contradiction (BDR). It is obvious from results that the talked about methodology has significant improvement in BDR at low SNR by applying the chose window size. The presentation of the talked about methodology is additionally assessed by performing irregularity testing of the created keys utilizing NIST test set for haphazardness. Wang, Wei Et al. [14] authors investigate a secure cloud-assisted IoT 
data managing method to keep data confidentiality when collecting, storing and accessing IoT data with the assistance of a cloud with the consideration of users' increment. The discussed system novel applies a proxy re-encryption scheme, which was discussed. Hence, a secure IoT under their discussed method could resist most attacks from both insiders and outsiders of IoT to break data confidentiality, and meanwhile with constant communication cost for re-encryption anti incremental scale of IoT. authors further show the method is practical by numerical results. Yang, Yang Et al. [15] a protection safeguarding savvy IoT-based medical care huge information stockpiling framework with self-versatile access control is talked about. The point is to guarantee the security of patients' medical care information, acknowledge access control for typical and crisis situations, and backing shrewd deduplication to save the extra room in large information stockpiling framework. The clinical files created by the medical services IoT network are scrambled and moved to the capacity framework, which can be safely divided between the medical care staff from various clinical do-mains utilizing a cross-space access control strategy. The customary access control innovation permits the approved information clients to decode patient's delicate clinical information, yet additionally hampers the firsthelp therapy when the patient's life is compromised on the grounds that the on location first-help staff are not allowed to get patient's authentic clinical information. Zhao, Quanyu Et al. [16] authors utilize the blockchain to construct a novel privacy-preserving remote data integrity checking scheme for IoT information management systems without involving trusted third parties. Their scheme leverages the Lifted EC-ElGamal cryptosystem, bilinear pairing, and blockchain to support efficient public batch signature verifications and protect the security and data privacy of the IoT systems. The results of the experiment demonstrate the efficiency of their scheme. Stergiou, Christos Et al. [17] authors present a survey of IoT and Cloud Computing with a focus on the security issues of both technologies. Specifically, authors combine the two aforementioned technologies in order to examine the common features, and in order to discover the benefits of their integration. Concluding, authors present the contribution of Cloud Computing to the IoT technology. Thus, it shows how the Cloud Computing technology improves the function of the IoT. Finally, authors survey the security challenges of the integration of IoT and Cloud Computing. Mohanty, Sachi Nandan Et al. [18] ELIB model is created to meet requires of IoT. The introduced model is sent in a brilliant home climate as a significant outline to confirm its pertinence in different IoT situations. The asset obliged assets in a keen home takes the points of interest from a brought together chief which creates shared keys to communicate information, measure each approaching and active solicitations. The introduced ELIB model creates an overlay network where profoundly prepared assets can converge to a public BC which checks committed security and protection. A bunch of three enhancements are completed in the introduced ELIB model incorporate lightweight agreement calculation, CC cryptography and DTM conspire. A point-by-point recreation happens under various situations as far as preparing time, energy utilization and overhead. The ELIB accomplishes a sum of half saving in handling time on contrasting with gauge technique with the base energy utilization of $0.07 \mathrm{~mJ}$. The got trial result demonstrated that the ELIB shows greatest execution under a few assessment boundaries. Chatterjee, Baibhab Et al. [19] Traditional confirmation in radio-recurrence $(\mathrm{RF})$ frameworks empower secure information correspondence inside an organization through procedures, for example, advanced marks and HMAC, which experience the ill effects of keyrecuperation assaults. Cutting edge IoT organizations, for example, Nest additionally use OAuth 2.0 conventions that are powerless against CSRF, which shows that these procedures may not keep an enemy from replicating or demonstrating the mystery IDs or encryption keys utilizing obtrusive, side channel, learning or programming assaults. PUF, then again, can abuse fabricating measure varieties to particularly distinguish silicon chips which makes a PUF-based framework very vigorous and secure requiring little to no effort, as it is basically difficult to duplicate similar silicon attributes across passes on. Taking motivation from human correspondence, which uses intrinsic varieties in the voice marks to distinguish a specific speaker, creators present RF-PUF: a profound neural organization-based system that permits constant confirmation of remote hubs, utilizing the impacts of innate cycle minor departure from RF properties of the remote $\mathrm{Tx}$, recognized through in-situ $\mathrm{AI}$ at the $\mathrm{Rx}$ end. The examined strategy uses the generally existing deviated RF correspondence structure and doesn't need any extra hardware for PUF age or highlight extraction. The weight of gadget ID is totally moved to the passage $\mathrm{Rx}$, like the activity of a human audience's cerebrum. Reenactment results including the cycle varieties in a standard $65 \mathrm{~nm}$ innovation hub, and highlights, for example, LO balance and I-Q unevenness recognized with a neural organization having 50 neurons in the shrouded layer demonstrate that the structure can 
recognize up to 4800 transmitters with a precision of 99.9\% under shifting channel conditions, and without the requirement for conventional prefaces. The examined plan can be utilized as an independent security include, or as a piece of conventional multifaceted verification. Korenda, Ashwija Reddy Et al. [20] The fuzzy extractor discussed in this paper ensures much less mutual information between the generated keys and the helper data. The experimental results show that their discussed scheme is capable of generating notably stronger keys compared to existing techniques, while utilizing a significantly lower number of helper data bits. Jiao, Long, Ning Wang Et al. [21] The blossom of the 5G correspondence and past fills in as an impetus for actual layer key age strategies. In 5G interchanges frameworks,

of actual layer key age in 5G and past interchanges are examined. Meneghello, Francesca Et al. [22] The IoT is quickly spreading, arriving at a large number of various spaces, including individual medical care, ecological checking, home mechanization, savvy portability, and Industry 4.0. As an outcome, increasingly more IoT gadgets are being conveyed in an assortment of public and private conditions, logically turning out to be normal objects of regular day to day existence. It is consequently evident that, in such a situation, online protection gets basic to stay away from dangers like spillage of reasonable data, forswearing of administration assaults, unapproved network access, etc. Tragically, some lowend IoT business items don't for the most part uphold solid security instruments, and can subsequently be focus of - or even methods for - various security assaults. The point of this paper is to give an expansive outline of the security hazards in the IoT area and to examine some potential balances. To this end, after an overall prologue to security in the IoT area, creators examine the particular security systems embraced by the most famous IoT correspondence conventions. At that point, creators report and investigate a portion of the assaults against genuine IoT gadgets revealed in the writing, to bring up the current security shortcomings of business IoT arrangements and comment the significance of thinking about security as an essential part in the plan of IoT frameworks. creators close the paper with a contemplated examination of the considered IoT innovations concerning a bunch of qualifying security credits, in particular honesty, obscurity, classification, protection, access control, verification, approval, versatility, self-association. Mama, Mingxin Et al. [23] The fast improvement of the IoT and the hazardous development of important information created by client hardware have prompted solid interest for access control, particularly progressive access control, which is numerous difficulties in conventional actual layer key age plans, for example, co-found busybodies, the high piece contradiction proportion, and high worldly relationship, could be survived. This paper records the key-empowering influence strategies in $5 \mathrm{G}$ remote organizations, which offer occasions to address existing issues in actual layer key age. creators overview the current key age strategies and present potential answers for the current issues. The new arrangements incorporate applying the high sign directionality in beamforming to oppose co-found snoops, using the sparsity of mmWave channel to accomplish a low piece difference proportion under low SNR, and abusing cross breed precoding to diminish the fleeting relationship among estimated tests. At long last, the future patterns

performed from a gathering correspondence point of view. Nonetheless, the key administration systems for a particularly future Internet depend generally on a confided in outsider that requires full trust of the KGC or CA. Late examinations show that brought together cloud habitats will be probably not going to convey palatable administrations to clients since creators place a lot of trust in outsiders; accordingly, these focuses don't matter to client protection arranged situations. This paper tends to these issues by examined a novel BDKMA with haze processing to decrease idleness and multiblock chains worked in the cloud to accomplish cross-space access. The talked about plan uses blockchain innovation to fulfill the decentralization, fine-grained auditability, high adaptability, and extensibility prerequisites, just as the protection saving standards for progressive access control in IoT. creators planned framework tasks strategies and presented diverse approval task modes and gathering access examples to strengthen the extensibility. creators assessed the presentation of their examined design and contrasted it and existing models utilizing different execution measures. The reproduction results show that the multiblock chain structure generously improves framework execution, and the versatility is brilliant as the organization size increments. Besides, dynamic exchange assortment time change empowers the presentation and framework ability to be improved for different conditions. Luo, Entao Et al. [24] creators initially explore the difficulties with security ensured information assortment. At that point creators talked about a functional system called Privacy-Protector, understanding security ensured information assortment, with the target of forestalling these sorts of assaults. Security Protector incorporates the thoughts of mystery sharing and offer fixing for patients' information security. Since it is the first run through, creators apply the SW-SSS in Privacy-Protector. In the system, creators 
utilize a disseminated data set comprising of numerous cloud workers, which guarantees that the security of patients' very own information can stay ensured up to one of the workers remains positive. creators additionally present a patient access control plot in which numerous cloud workers team up in shared development to offer patients' information to medical services suppliers without uncovering the substance of the information. The security execution investigation has indicated that the Privacy-Protector system is secure and security ensured against different assaults. Li, Guyue Et al. [25] The 5G and past remote correspondences will change many energizing applications and trigger gigantic information associations with private, secret, and touchy data. The security of remote interchanges is routinely settled by cryptographic plans and conventions in which the mystery key dispersion is one of the fundamental natives. Nonetheless, customary cryptography-based key conveyance conventions may be tested in the $5 \mathrm{G}$ and past interchanges in light of extraordinary highlights, for example, gadget to-gadget and heterogeneous correspondences, and super low inertness prerequisites. CRKG is an arising actual layer-based strategy to build up mystery keys between gadgets. This article surveys CRKG when the $5 \mathrm{G}$ and past organizations utilize three applicant advances: duplex modes, MIMO and mmWave correspondences. Creators recognize the chances and difficulties for CRKG and give relating arrangements. To additionally show the achievability of CRKG in commonsense correspondence frameworks, creators review existing models with various IoT conventions and inspect their exhibition in certifiable conditions. This article shows the attainability and promising exhibitions of CRKG with the possibility to be marketed. Huang, Junqin Et al. [26] IIoT assumes an in-nonessential part for Industry 4.0, where individuals are submitting ted to execute a general, adaptable, and secure IIoT framework to be embraced across different enterprises. In any case, existing IIoT frameworks are helpless against single purpose of disappointment and malignant assaults, which can't offer stable types of assistance. Because of the flexibility and security guarantee of blockchain, consolidating blockchain and IoT acquires impressive interest. Nonetheless, blockchains are power-serious and low-throughput, which are not reasonable for powercompelled IoT gadgets. To handle these difficulties, creators present a blockchain framework with creditbased agreement instrument for IIoT. creators talked about a credit-based PoW component for IoT gadgets, which can ensure framework security and exchange efficiency at the same time. To secure delicate information confidentiality, creators plan an information authority the board strategy to manage the admittance to sensor information. Moreover, their framework is constructed dependent on coordinated non-cyclic chart organized blockchains, which is more efficient than the Satoshi-style blockchain in execution. creators execute the framework on Raspberry $\mathrm{Pi}$, and direct a contextual analysis for the brilliant processing plant. Broad assessment and investigation results show that creditbased PoW component and information access control are secure and efficient in IIoT.Aman, Muhammad Naveed Et al. [27] Devices in the IoT produce a lot of touchy information. Nonetheless, the utilization of the public Internet for information move by IoT gadgets makes them defenseless to digital assaults. Among these assaults, information altering or adjustment at-tacks to disturb or predisposition the conditions of uses utilizing these information may bring about boundless harm and blackouts. To distinguish such assaults, this paper examined a productive and straightforward method to identify information altering in IoT frameworks. The examined component utilizes an irregular time bouncing arrangement and arbitrary stages to shroud approval data. creators additionally present a conventional security examination of the talked about convention. Execution examination of the talked about convention shows that it has low computational multifaceted nature and is reasonable for IoT frameworks. Wang, Ning Et al. [28] The $5 \mathrm{G}$ remote advances fill in as a critical propellent to satisfy the expanding needs of things to come IoT organizations. For remote correspondence security in $5 \mathrm{G}$ IoT organizations, PLS has as of late got developing interest. This article intends to give a far-reaching overview of the PLS procedures in 5G IoT correspondence frameworks. The examination comprises of four various leveled parts. In the first part, creators survey the qualities of 5G IoT under ordinary application situations. creators at that point present the security dangers from the 5G IoT actual layer and order them as per the various motivations behind the assailant. In the third part, creators look at the $5 \mathrm{G}$ correspondence advancements in 5G IoT frameworks and examine their difficulties and openings when adapting to physical-layer dangers, including monstrous MIMO, mmWave interchanges, NOMA, full-duplex innovation, EH, VLC and UAV interchanges. At long last, creators examine open exploration issues and future works about PLS in the IoT framework with advances of $5 \mathrm{G}$ and past. Abbas, Nadeem Et al. [29] IoT is an arising worldview marked by heterogeneous advancements made out of keen universal articles that are consistently associated with the Internet. These articles are sent as LLN to offer inventive types of assistance in different application spaces, for 
example, brilliant urban areas, shrewd wellbeing, and keen networks. The LLN is a type of an organization where the interconnected gadgets are exceptionally asset compelled and portrayed by high misfortune rates, low information rates, and unsteadiness in the correspondence joins. Also, IoT gadgets produce a gigantic measure of secret and security-touchy information. Different cryptographic-based methods exist that can adequately adapt to security assaults however are not reasonable for IoT as they bring about maximum usage of assets. One approach to address this issue is by offloading the extra security-related activities to a more creative substance, for example, a mist-based hub. By and large, mist registering empowers security and investigation of dormancy touchy information straightforwardly at the organization's edge. This paper examined a novel FSS to give start to finish security at the haze layer for IoT gadgets utilizing two grounded cryptographic plans, character-based encryption, and personality-based mark. The FSS gives security administrations, for example, verification, privacy, and non-disavowal. The talked about engineering would be actualized and assessed in an OPNET test system utilizing a solitary organization geography with various traffic loads. The FSS performed better when contrasted and the APaaS and the heritage strategy. Moosavi, Sanaz Rahimi Et al. [30] Authors break down the presentation of the best in class start to finish security plans in medical care IoT frameworks. creators recognize that the fundamental necessities of powerful security answers for medical services IoT frameworks involve low inactivity secure key age approach utilizing patients' ECG signals, secure and productive confirmation and approval for medical services IoT gadgets dependent on the authentication based DTLS, and strong and secure portability empowered start to finish correspondence dependent on DTLS meeting resumption. The exhibition of the cutting-edge security arrangements including their start to finish security conspire is tried by building up a model medical care IoT framework. The model is worked of a Pandaboard, a TI SmartRF06 board and WiSMotes. The Pandaboard alongside the CC2538 module goes about as a brilliant entryway and the WisMotes go about as clinical sensor hubs. In view of the investigation, creators discovered that their answer has the broadest arrangement of execution highlights in contrast with related methodologies found in the writing. III.

\section{CRYPTOGRAPHY AND KEY GENERATION APPROACH}

The exhibition assessment results show that contrasted with the current methodologies, the cryptographic key age approach examined in their start to finish security conspire is on normal 1.8 occasions quicker than existing key age draw near while being more energy-productive. Badr, Shaimaa Et al. [31] to meet the prerequisite of dispersed design in the EHRs framework, creators examined a novel convention to accomplish an ideal protection saving for the patient in particular PBE-DA by applying the idea of Blockchain on the medical care correspondence substances in an e-wellbeing stage. Consequently, PBE-DA will be utilized to help the patient namelessly to access, check or update his delicate information on EHRs framework. Additionally, creators broke down not just the public blockchain level between the distinctive EHRs cloud supplier yet additionally another Blockchain level between the patient sensors and the patient framework as a door for the entire medical services stage. El-Hajj, Mohammed Et al. [32] The IoT is the capacity to furnish regular gadgets with a method of ID and another path for correspondence with one another. The range of IoT application areas is exceptionally enormous including savvy homes, shrewd urban communities, wearables, e-wellbeing, and so on Thus, tens and even many billions of gadgets will be associated. Such gadgets will have savvy abilities to gather, investigate and even settle on choices with no human association. Security is an incomparable prerequisite in such conditions, and specifically verification is of high interest given the harm that could occur from a noxious unauthenticated gadget in an IoT framework. This paper gives a close to finish and cuttingedge perspective on the IoT validation field. It gives a synopsis of an enormous scope of validation conventions examined in the writing. Utilizing a multi-measure order recently presented in their work, it thinks about and assesses the examined validation conventions, indicating their qualities and shortcomings, which establishes an essential initial step for scientists and engineers tending to this space. Zikria, Yousaf Bin Et al. [33] authors present brief overview of different IoT OSs, supported hardware, and future research directions. Therein, authors provide overview of the accepted papers in their Special Issue on IoT OS management: opportunities, challenges, and solution. Finally, authors conclude the manuscript.

The cryptography is a mathematical formation and derivation of application in terms of change the value and attribute of data. the changing behaviors of mathematics is called cryptography, the two major terms used in 
cryptography is encryption and decryption. In the process of encryption and decryption involves the value of key[1, 19]. This value of key depends on the approach of algorithms, the approach of algorithms deals in manners of symmetric, asymmetric and hybrid cryptography. the symmetric cryptography is also called private cryptography, in this only one key is used for the encryption and decryption[20]. Instead of asymmetric cryptography is also called public cryptography, this method applied two different keys for the process of encryption of description. Some other cryptography algorithms is based on the combination of public and private key cryptography[21, 22].

In concern of security strength, the public key cryptography is strong instead of private cryptography. But the limitation of public cryptography is maximum number of iteration and required extra memory space for the processing of key data during the process of session, most of tiny device avoid the application of public cryptography[23, 24]. The most of IoT enable device utilize private cryptography methods along with message digest (MD). The message digest algorithm provides the hash function capacity of key. The value of key generation along with hash code is robust. The hash code along message is tampered, the size of message and key are change. The vary famous hash code algorithm is SHA-1, SHA-3 and SHA-5 other also applied MD1 to MD5 digest algorithm for the generation of hash code for the session and generation of key[25].

In IoT enable device, the communication between nodes and the infrastructure needs light key distribution methods using the public key to reduce cost trade off, now it is difficult to apply the methods to IoT device where encryption module, such as AES (advance encryption standard), RSA, ECC cannot be mounted. The other key generation methods use transform based function. The transform-based function is very efficient for the generation of key for IoT based communication. the mainly use two transform function for the generation of key is DCT (discrete cosine transform) and DWT (discrete wavelet transform)[26, 27, 28]. The sensing strength of these transform function is quite good instead of conventional cryptography methods.

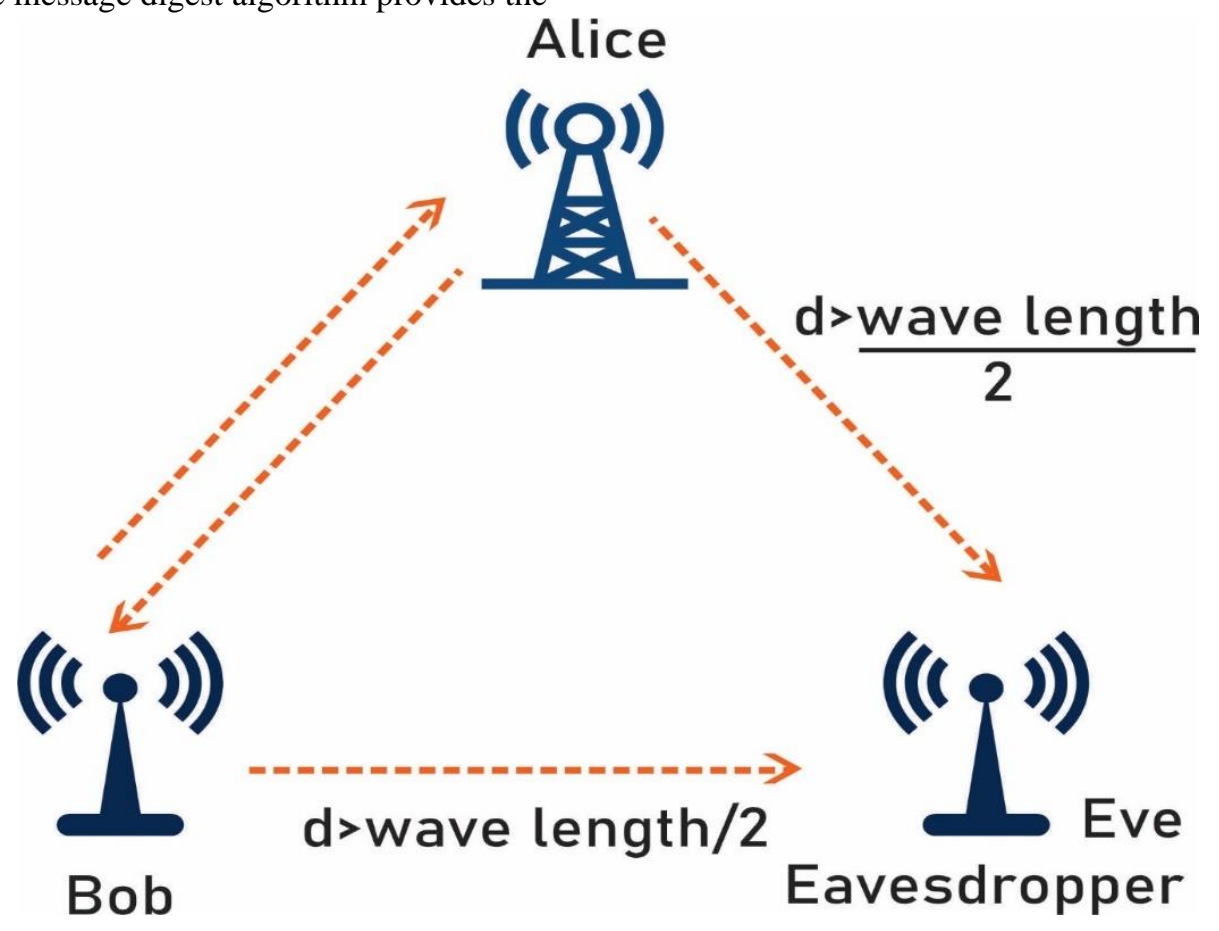

Figure 1: Process of communication apply in IoT enabled device, Alice and Bob is two authenticated user and Eve is third part man in middle attack.

The figure 1 show the system model of IoT enable communication system. The main two party share the value of key for the process of communication. the Eve is third party act as man in middle attack, the man in middle attack senses the signal strength and break the link of communication and theft of data for the processing of unauthorized access. For this challenge and issue the IoT enable communication devices derive dynamic key generation methods and enhance the security of IoT enable devices.

\section{ANALYSIS OF KEY}

To evaluate the performance of cryptography algorithm for the process of encryption and decryption applied text 
data file with variable size and key length. In the process of analysis applied mainly these algorithms AES, DES, RSA, ECC, DCT and DWT. For the implementation purpose use MATLAB software and core I7 process with windows 10 operating system. The evaluation of parameters considers of computational time and energy factor during the generation of key[29, 30, 31, 32].

\begin{tabular}{|c|c|c|}
\hline Technique & Energy Factor(Joule) & Completion Time $(m s)$ \\
\hline AES[34] & 1.33 & 8.040 \\
\hline DES[35] & 0.92 & 7.012 \\
\hline RSA[36] & 0.88 & 7.955 \\
\hline DCT[37] & 1.25 & 7.215 \\
\hline DWT[38] & 1.12 & 6.586 \\
\hline ECC[39] & 1.56 & \\
\hline
\end{tabular}

Table 1: Result analysis of different techniques AES, DES, RSA, DCT, DWT and ECC using 25kb file size.

\begin{tabular}{|c|c|c|}
\hline Technique & Energy Factor(Joule) & Completion Time $(m s)$ \\
\hline AES[34] & 0.95 & 6.476 \\
\hline DES[35] & 1.63 & 7.328 \\
\hline RSA[36] & 1.56 & 6.128 \\
\hline DCT[37] & 0.95 & 6.057 \\
\hline DWT[38] & 0.98 & 5.589 \\
\hline ECC[38] & 1.81 & . \\
\hline
\end{tabular}

Table 2: Result analysis of different techniques AES, DES, RSA, DCT, DWT and ECC using 50kb file size.

\begin{tabular}{|c|c|c|}
\hline Technique & Energy Factor(Joule) & Completion Time $(m s)$ \\
\hline AES[34] & 0.90 & 9.036 \\
\hline DES[35] & 0.84 & 9.773 \\
\hline RSA[36] & 1.11 & 8.193 \\
\hline DCT[37] & 1.34 & 8.641 \\
\hline DWT[38] & 1.47 & 7.246 \\
\hline ECC[39] & 1.68 & \\
\hline
\end{tabular}

Table 3: Result analysis of different techniques AES, DES, RSA, DCT, DWT and ECC using 100kb file size.

\begin{tabular}{|c|c|c|}
\hline Technique & Energy Factor(Joule) & Completion Time $(m s)$ \\
\hline AES[34] & 1.53 & 4.322 \\
\hline DES[35] & 1.46 & 5.337 \\
\hline
\end{tabular}




\begin{tabular}{|c|c|c|}
\hline RSA[36] & 1.26 & 5.591 \\
\hline DCT[37] & 1.14 & 4.389 \\
\hline DWT[38] & 1.35 & 4.679 \\
\hline ECC[39] & 1.72 & 4.004 \\
\hline
\end{tabular}

Table 4: Result analysis of different techniques AES, DES, RSA, DCT, DWT and ECC using 250kb file size.

\begin{tabular}{|c|c|c|}
\hline Technique & Energy Factor(Joule) & Completion Time(ms) \\
\hline AES[34] & 0.80 & 5.478 \\
\hline DES[35] & 0.87 & 7.940 \\
\hline RSA[36] & 0.76 & 6.079 \\
\hline DCT[37] & 0.87 & 7.233 \\
\hline DWT[38] & 1.18 & 4.652 \\
\hline ECC[39] & 1.24 & 4.018 \\
\hline
\end{tabular}

Table 5: Result analysis of different techniques AES, DES, RSA, DCT, DWT and ECC using 500kb file size.

\begin{tabular}{|c|c|c|}
\hline Technique & Energy Factor(Joule) & Completion Time(ms) \\
\hline AES[34] & 0.85 & 9.808 \\
\hline DES[35] & 0.80 & 10.993 \\
\hline RSA[36] & 0.73 & 8.163 \\
\hline DCT[37] & 0.91 & 9.873 \\
\hline DWT[38] & 0.98 & 8.642 \\
\hline ECC[39] & 1.27 & 7.685 \\
\hline
\end{tabular}

Table 6: Result analysis of different techniques AES, DES, RSA, DCT, DWT and ECC using 1000kb file size. 


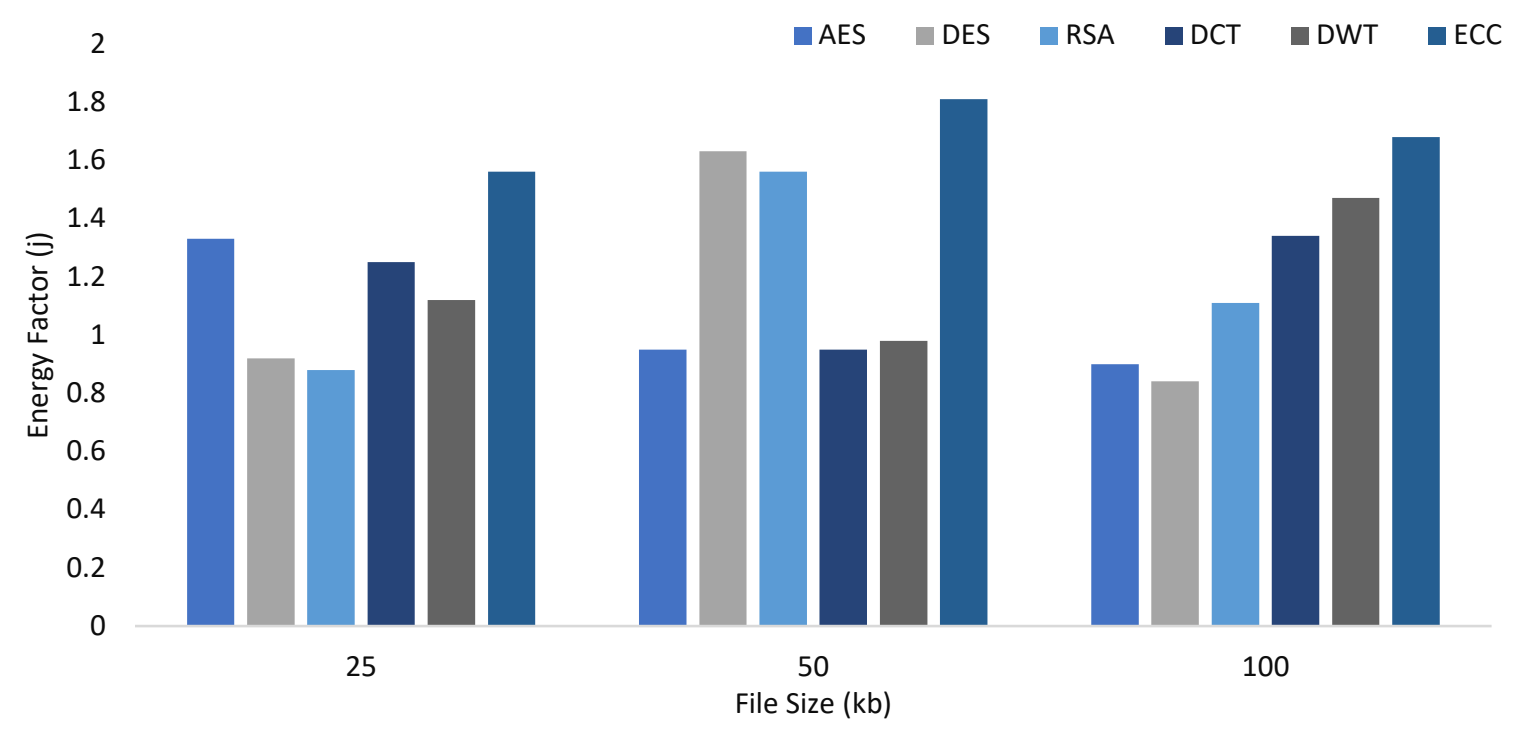

Figure 2: Performance analysis of energy factor (joule) the different cryptography techniques AES[34], DES[35], RSA[36], DCT[37], DWT[38] and ECC[39] using 25kb, 50kb, 100kb file sizes in our simulation model analysis.

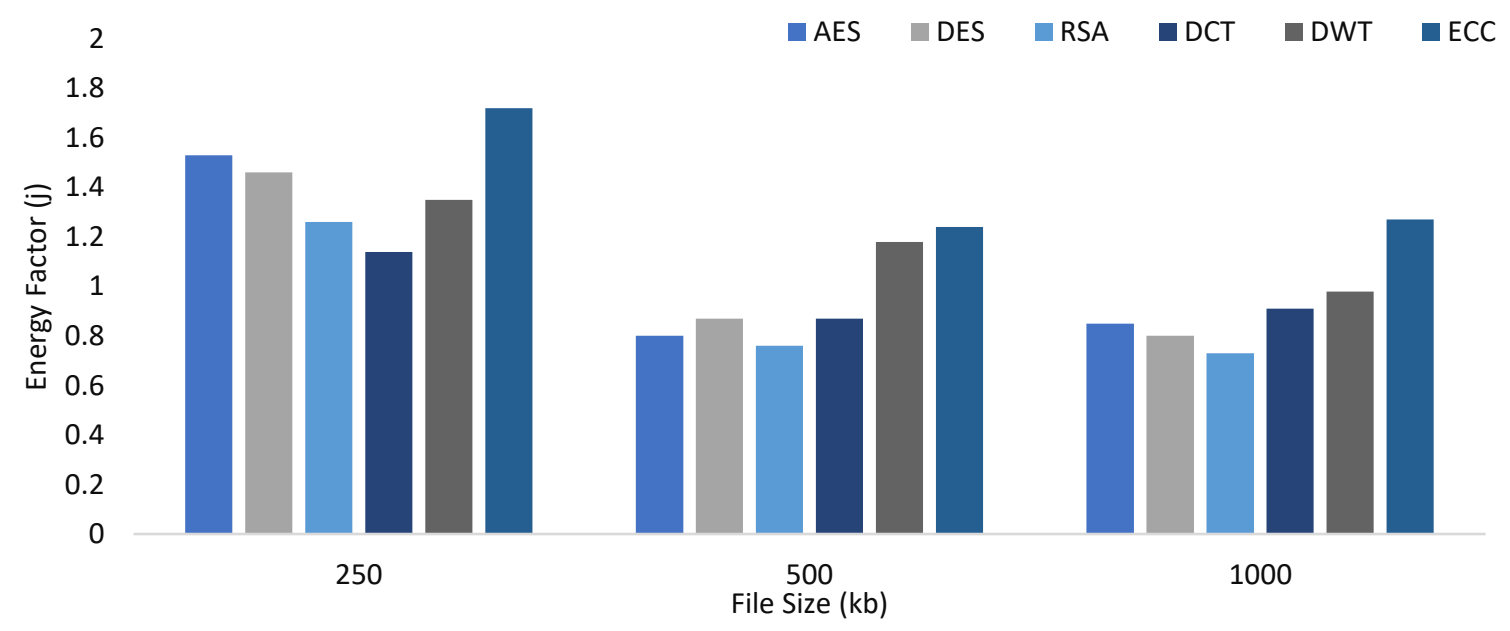

Figure 3: Performance analysis of energy factor (joule) the different cryptography techniques AES[34], DES[35], RSA[36], DCT[37], DWT[38] and ECC[39] using 25kb, 50kb, 100kb file sizes in our simulation model analysis.
12
$\square \mathrm{AES} \quad \mathrm{DES} \quad \mathrm{RSA} \quad \square \mathrm{DCT} \quad \square \mathrm{DWT} \quad \square \mathrm{ECC}$

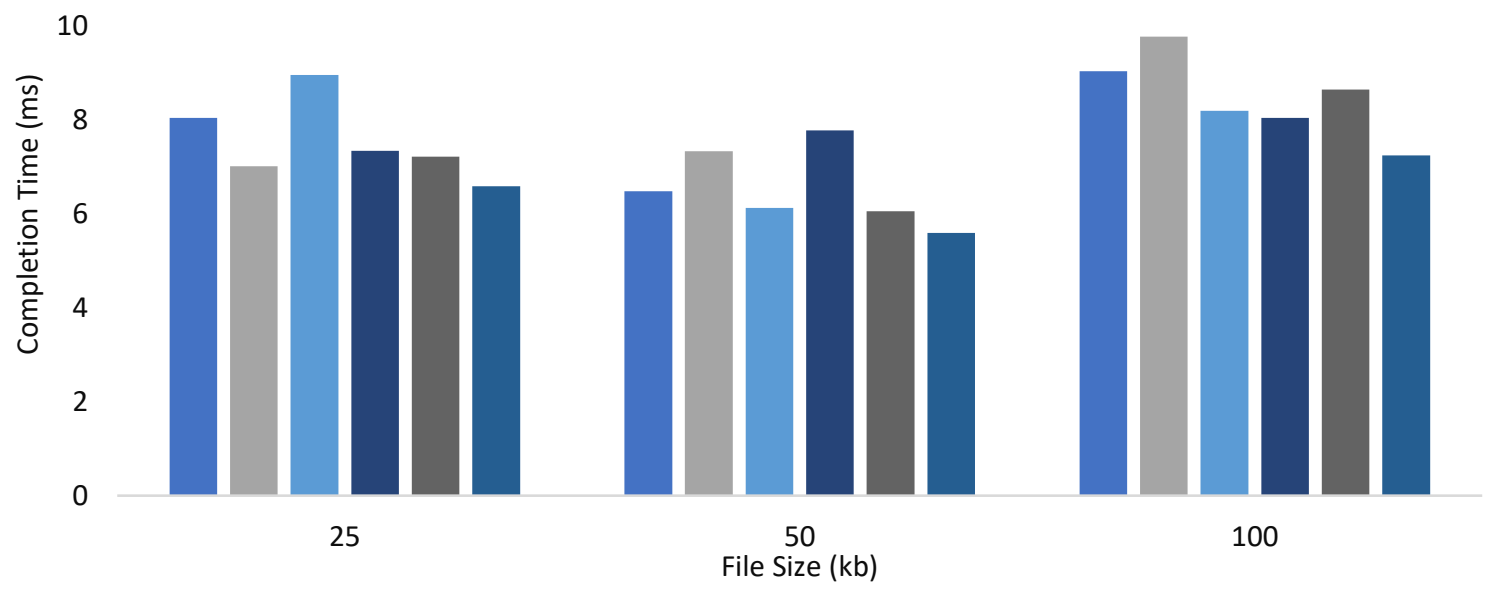


Figure 4: Performance analysis of completion time (ms) the different cryptography techniques AES[34], DES[35], RSA[36], DCT[37], DWT[38] and ECC[39] using 25kb, 50kb, 100kb file sizes in our simulation model analysis.

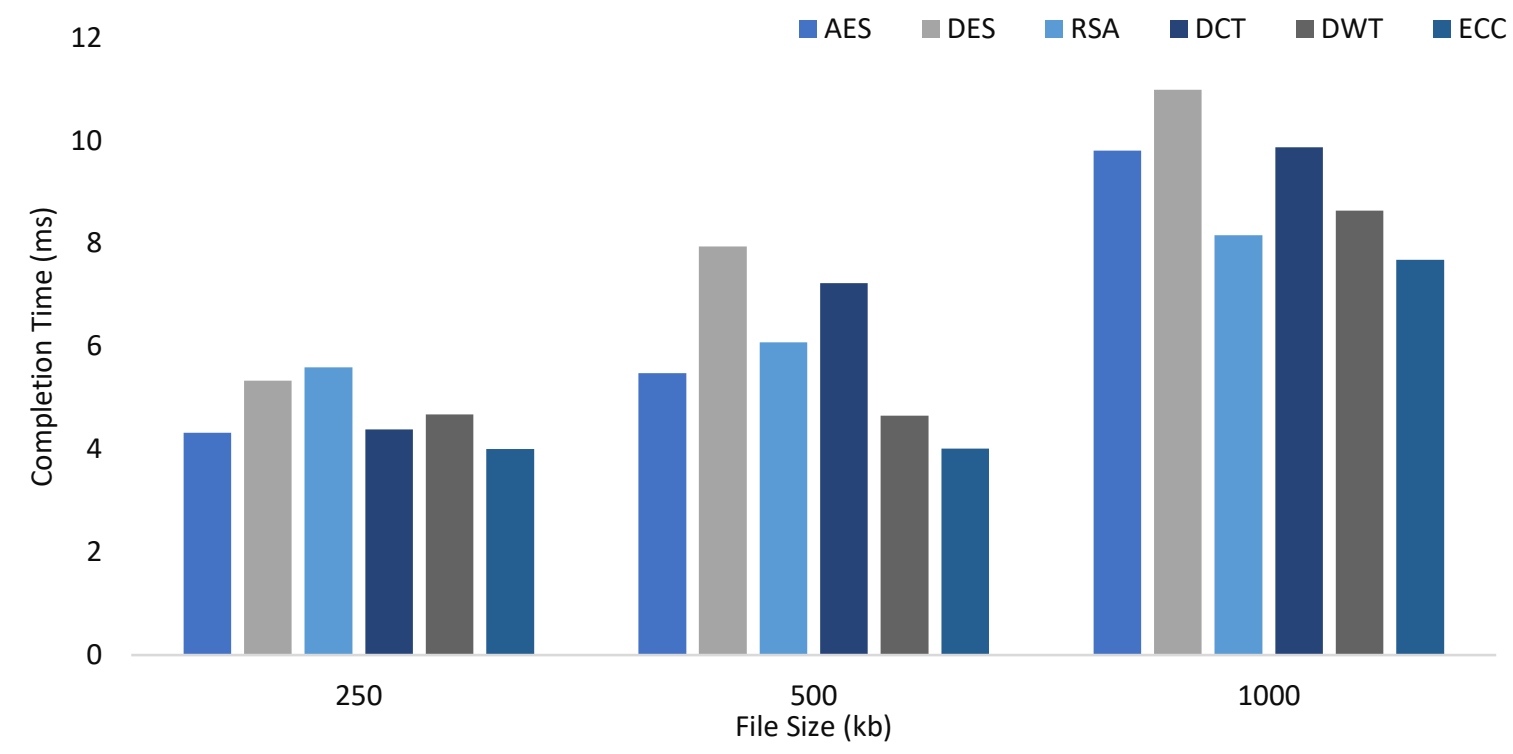

Figure 5: Performance analysis of completion time (ms) the different cryptography techniques AES[34], DES[35], RSA[36], DCT[37], DWT[38] and ECC[39] using 25kb, 50kb, 100kb file sizes in our simulation model analysis.

\section{CONCLUSION \& FUTURE SCOPE}

The reachability of IoT enabled devices in every filed of society. The vulnerable communication protocol of embedded security protocol cannot satisfy wireless communication's security goals, and IoT enabled devices. The study finds some severe threats regarding the security hole of key generation and key distribution. The conventional cryptography algorithms cannot handle the security issue, and key generation mechanism of IoT enable devices. The open communication protocol stack needs interpretability with the standard protocol of a wireless sensor network. This paper analysed the efficiency of energy consumption and computational efficiency of public, private and some other key generation algorithms such as DCT and DWT. The computational complexity of high, but the strength of the key is also high. However, the limitation of memory and bandwidth needs efficient light key generation methods. The dynamic and non-conventional key generation algorithm, such as DCT and DWT is perfect for computational complexity. These algorithm methods encapsulated with the physical layer and applied for modulation and key generation. These process of key generation proposed new direction of authentication and data integrity for IoT enabled devices. For future work, derived another key generation algorithm for IoT enabled devices. The derived algorithm tested on the real information environments such as tampering and man in the middle attack to theft the information and countermeasure the key generation algorithm's security strength.

\section{REFERENCES}

[1]. Khalid, Umair, Muhammad Asim, Thar Baker, Patrick CK Hung, Muhammad Adnan Tariq, and Laura Rafferty. "A decentralized lightweight blockchain-based authentication mechanism for IoT systems." Cluster Computing (2020): 1-21.

[2]. Sobin, C. C. "A survey on architecture, protocols and challenges in iot." Wireless Personal Communications 112, no. 3 (2020): 1383-1429.

[3]. Cao, Jin, Pu Yu, Xinyin Xiang, Maode Ma, and Hui Li. "Anti-quantum fast authentication and data transmission scheme for massive devices in 5G NBIoT system." IEEE Internet of Things Journal 6, no. 6 (2019): 9794-9805.

[4]. Chen, Bin, and Frans MJ Willems. "Secret key generation over biased physical unclonable functions with polar codes." IEEE Internet of Things Journal 6, no. 1 (2018): 435-445.

[5]. Deng, Lianbing, Daming Li, Xiang Yao, David Cox, and Haoxiang Wang. "Mobile network intrusion detection for IoT system based on transfer learning algorithm." Cluster Computing 22, no. 4 (2019): 9889-9904.

[6]. Fan, Kai, Huiyue Xu, Longxiang Gao, Hui Li, and Yintang Yang. "Efficient and privacy preserving 
access control scheme for fog-enabled IoT." Future Generation Computer Systems 99 (2019): 134-142.

[7]. Hamza, Rafik, Zheng Yan, Khan Muhammad, Paolo Bellavista, and Faiza Titouna. "A privacypreserving cryptosystem for IoT Ehealthcare." Information Sciences 527 (2020): 493510.

[8]. Lee, Joohee, Duhyeong Kim, Hyungkyu Lee, Younho Lee, and Jung Hee Cheon. "RLizard: Postquantum key encapsulation mechanism for IoT devices." IEEE Access 7 (2018): 2080-2091.

[9]. Nausheen, Farha, and Sayyada Hajera Begum. "Healthcare IoT: benefits, vulnerabilities and solutions." In 2018 2nd International Conference on Inventive Systems and Control (ICISC), pp. 517522. IEEE, 2018.

[10]. Qu, Chao, Ming Tao, Jie Zhang, Xiaoyu Hong, and Ruifen Yuan. "Blockchain based credibility verification method for IoT entities." Security and Communication Networks 2018 (2018).

[11]. Shah, Trusit, and Subbarayan Venkatesan. "Authentication of IoT device and IoT server using secure vaults." In 2018 17th IEEE International Conference On Trust, Security And Privacy In Computing And Communications/12th IEEE International Conference On Big Data Science And Engineering (TrustCom/BigDataSE), pp. 819-824. IEEE, 2018.

[12]. Song, Yujiao, Hao Wang, Xiaochao Wei, and Lei Wu. "Efficient attribute-based encryption with privacy-preserving key generation and its

[19]. Chatterjee, Baibhab, Debayan Das, Shovan Maity, and Shreyas Sen. "RF-PUF: Enhancing IoT security through authentication of wireless nodes using in-situ machine learning." IEEE Internet of Things Journal 6, no. 1 (2018): 388-398.

[20]. Korenda, Ashwija Reddy, Fatemeh Afghah, and Bertrand Cambou. "A secret key generation scheme for internet of things using ternary-states ReRAMbased physical unclonable functions." In 2018 14th International Wireless Communications \& Mobile Computing Conference (IWCMC), pp. 1261-1266. IEEE, 2018.

[21]. Jiao, Long, Ning Wang, $\mathrm{Pu}$ Wang, Amir Alipour-Fanid, Jie Tang, and Kai Zeng. "Physical layer key generation in $5 \mathrm{G}$ wireless networks." IEEE Wireless Communications 26, no. 5 (2019): 48-54.

[22]. Meneghello, Francesca, Matteo Calore, Daniel Zucchetto, Michele Polese, and Andrea Zanella. "IoT: Internet of threats? A survey of practical application in industrial cloud." Security and Communication Networks 2019 (2019).

[13]. Soni, Ankit, Raksha Upadhyay, and Abhay Kumar. "Wireless physical layer key generation with improved bit disagreement for the internet of things using moving window averaging." Physical Communication 33 (2019): 249-258.

[14]. Wang, Wei, Peng Xu, and Laurence Tianruo Yang. "Secure data collection, storage and access in cloud-assisted IoT." IEEE cloud computing 5, no. 4 (2018): 77-88.

[15]. Yang, Yang, Xianghan Zheng, Wenzhong Guo, Ximeng Liu, and Victor Chang. "Privacy-preserving smart IoT-based healthcare big data storage and selfadaptive access control system." Information Sciences 479 (2019): 567-592.

[16]. Zhao, Quanyu, Siyi Chen, Zheli Liu, Thar Baker, and Yuan Zhang. "Blockchain-based privacy-preserving remote data integrity checking scheme for IoT information systems." Information Processing \& Management 57, no. 6 (2020): 102355.

[17]. Stergiou, Christos, Kostas E. Psannis, ByungGyu Kim, and Brij Gupta. "Secure integration of IoT and cloud computing." Future Generation Computer Systems 78 (2018): 964-975.

[18]. Mohanty, Sachi Nandan, K. C. Ramya, S. Sheeba Rani, Deepak Gupta, K. Shankar, S. K. Lakshmanaprabu, and Ashish Khanna. "An efficient Lightweight integrated Blockchain (ELIB) model for IoT security and privacy." Future Generation Computer Systems 102 (2020): 1027-1037.

security vulnerabilities in real IoT devices." IEEE Internet of Things Journal 6, no. 5 (2019): 81828201.

[23]. Ma, Mingxin, Guozhen Shi, and Fenghua Li. "Privacy-oriented blockchain-based distributed key management architecture for hierarchical access control in the IoT scenario." IEEE Access 7 (2019): 34045-34059.

[24]. Luo, Entao, Md Zakirul Alam Bhuiyan, Guojun Wang, Md Arafatur Rahman, Jie Wu, and Mohammed Atiquzzaman. "Privacyprotector: Privacy-protected patient data collection in IoTbased healthcare systems." IEEE Communications Magazine 56, no. 2 (2018): 163-168.

[25]. Li, Guyue, Chen Sun, Junqing Zhang, Eduard Jorswieck, Bin Xiao, and Aiqun Hu. "Physical layer key generation in $5 \mathrm{G}$ and beyond wireless communications: Challenges and opportunities." Entropy 21, no. 5 (2019): 497. 
[26]. Huang, Junqin, Linghe Kong, Guihai Chen, Min-You Wu, Xue Liu, and Peng Zeng. "Towards secure industrial IoT: Blockchain system with credit-based consensus mechanism." IEEE Transactions on Industrial Informatics 15, no. 6 (2019): 3680-3689.

[27]. Aman, Muhammad Naveed, Biplab Sikdar, Kee Chaing Chua, and Anwar Ali. "Low power data integrity in IoT systems." IEEE Internet of Things Journal 5, no. 4 (2018): 3102-3113.

[28]. Wang, Ning, Pu Wang, Amir Alipour-Fanid, Long Jiao, and Kai Zeng. "Physical-layer security of $5 \mathrm{G}$ wireless networks for IoT: Challenges and opportunities." IEEE Internet of Things Journal 6, no. 5 (2019): 8169-8181.

[29]. Abbas, Nadeem, Muhammad Asim, Noshina Tariq, Thar Baker, and Sohail Abbas. "A mechanism for securing IoT-enabled applications at the fog layer." Journal of Sensor and Actuator Networks 8, no. 1 (2019): 16.

[30]. Moosavi, Sanaz Rahimi, Ethiopia Nigussie, Marco Levorato, Seppo Virtanen, and Jouni Isoaho. "Performance analysis of end-to-end security schemes in healthcare IoT." Procedia computer science 130 (2018): 432-439.

[31]. Badr, Shaimaa, Ibrahim Gomaa, and Emad Abd-Elrahman. "Multi-tier blockchain framework for IoT-EHRs systems." Procedia Computer Science 141 (2018): 159-166.

[32]. El-Hajj, Mohammed, Ahmad Fadlallah, Maroun Chamoun, and Ahmed Serhrouchni. "A survey of internet of things (IoT) authentication schemes." Sensors 19, no. 5 (2019): 1141.

[33]. Zikria, Yousaf Bin, Sung Won Kim, Oliver Hahm, Muhammad Khalil Afzal, and Mohammed Y. Aalsalem. "Internet of Things (IoT) operating systems management: Opportunities, challenges, and solution." (2019): 1793.

[34]. Tsai, Kun-Lin, Fang-Yie Leu, Ilsun You, ShuoWen Chang, Shiung-Jie Hu, and Hoonyong Park. "Low-Power AES Data Encryption Architecture for a LoRaWAN." IEEE Access 7 (2019): 146348146357.

[35]. Adhie, Roy Pramono, Yonatan Hutama, A. Saleh Ahmar, and M. I. Setiawan. "Implementation cryptography data encryption standard (DES) and triple data encryption standard (3DES) method in communication system based near field communication (NFC)." In Journal of Physics: Conference Series, vol. 954, no. 1, p. 012009. IOP Publishing, 2018.
[36]. Mahto, Dindayal, and Dilip Kumar Yadav. "Performance Analysis of RSA and Elliptic Curve Cryptography." IJ Network Security 20, no. 4 (2018): 625-635.

[37]. Jain, Yamini, Gaurav Sharma, Gaurav Anand, and Sangeeta Dhall. "A Hybrid Security Mechanism Based on DCT and Visual Cryptography for Data Communication Networks." In Cyber Security, pp. 131-142. Springer, Singapore, 2018.

[38]. Kaur, Kamal Nayan, Ishu Gupta, and Ashutosh Kumar Singh. "Digital image watermarking using $(2,2)$ visual cryptography with DWT-SVD based watermarking." In Computational intelligence in data mining, pp. 77-86. Springer, Singapore, 2019.

[39]. Rostampour, Samad, Masoumeh Safkhani, Ygal Bendavid, and Nasour Bagheri. "ECCbAP: A secure ECC-based authentication protocol for IoT edge devices." Pervasive and Mobile Computing 67 (2020): 101194. 